\title{
OBSERVATIONS OF ORION MOLECULAR CLOUD WITH NMA
}

\author{
Y. MURATA ${ }^{1}$, R. KAWABE ${ }^{2}$, M. ISHIGURO ${ }^{2}$, K.-I. MORITA ${ }^{2}$, \\ T. HASEGAWA ${ }^{3}$, M. HAYASHI ${ }^{4}$ \\ ${ }^{1}$ The Institute of Space and Astronautical Science \\ Sagamihara, 229, Japan,(E-mail murata@vsop.isas.ac.jp) \\ ${ }^{2}$ Nobeyama Radio Observatory, Minamimaki, Nagano, 384-13, Japan \\ ${ }^{3}$ The Institute of Astronomy, University of Tokyo, Mitaka, 181, Japan \\ ${ }^{4}$ Department of Astronomy, University of Tokyo, Bunkyo-ku, 113, Japan
}

\begin{abstract}
We have made aperture synthesis multifield observations of Orion Molecular Cloud-1 (OMC-1) in the CS (J=1-0) line using the Nobeyama Millimeter Array (NMA), and obtained $9^{\prime \prime}$ resolution maps over $10^{\prime}$ length. The OMC- 1 ridge shows a wiggled structure. The position angle of whole the ridge is $\sim 0^{\circ}-10^{\circ}$, but $\sim 20^{\circ}-30^{\circ}$ around the clumps. It is possible to make this structure by the magnetic field with a position angle of $\sim 150^{\circ}$. We also found filamentary structures in the northwest of Orion-KL, with a length-width ratio of more than 25 , which are made by the gas flow from Orion-KL.
\end{abstract}

\section{INTRODUCTION}

The OMC-1 ridge is an elongated cloud toward north-south direction with a length of $\sim 9^{\prime}$. It contains the Orion-KL region which appears as strong molecular lines and the continuum emission sources, and has been observed by a number of astronomers at millimeter and submillimeter wavelength. Orion-KL was mapped with various lines, and a wide area of OMC-1 with the Nobeyama Millimeter Array. The observations were carried out with higher velocity resolution, and higher dynamic range than previous observations to see the sub-structures of the clumps.

\section{OBSERVATIONS}

The CS $(1-0)$ transition $(48.990965 \mathrm{GHz}$ ) was observed toward 8 fields along the OMC-1 ridge. The data were obtained with a correlator bandwidth of $80 \mathrm{MHz}$, and a resolution of $78.1 \mathrm{kHz}\left(0.48 \mathrm{~km} \mathrm{~s}^{-1}\right)$. Each phase center was selected to overlap the circles of the primary beam width $\left(\sim 140^{\prime \prime}\right)$. The data were obtained from 1988 March to 1991 May in 3-6 configurations of the five-element interferometer. The typical noise level at each phase center is $150 \mathrm{mJy} / \mathrm{BEAM}$ ( 1 $\sigma)$. All field data were processed using AIPS and CNEANed with each CLEAN beam in natural weighting. The maps of the individual fields were then convolved to make all maps the same resolution $\left(8.5^{\prime \prime} \times 10.0^{\prime \prime}\right.$ with P.A. $\left.=20^{\circ}\right)$ and summed 
pixel-by-pixel with each pixel, in each field weighted. The individual field maps were cut off at $30 \%$ of the primary beam response, and only pixels with an accumulated primary beam response of $\geq 45 \%$ were kept in the final map.

\section{RESULTS}

Figure I shows a map integrated over a wide velocity range $\left(\mathrm{V}_{\mathrm{LSR}}=4.1-12.2\right.$ $\mathrm{km} \mathrm{s}^{-1}$ ). The emission from the OMC-1 ridge is oriented along the north-south direction from decl. $=-5^{\circ} 20^{\prime}$ to $-5^{\circ} 30^{\prime}$. The strongest emission peak at decl. $=$ $-5^{\circ} 24.5^{\prime}$ is Orion-KL. Long filamentary features ( $\mathrm{E}$ and $\mathrm{F}$ ) are also seen in the northwest of Orion-KL.

\section{Wiggled Structure of the OMC-1 ridge.}

The length of the ridge is $\lesssim 10^{\prime}$, and the width is $15^{\prime \prime}-40^{\prime \prime}$, which gives a ratio of length to width of $(12-32): 1$. The ridge shows three dominant clumps at decl.= $-5^{\circ} 22.6^{\prime},-5^{\circ} 24.5^{\prime}$, and $-5^{\circ} 26.2^{\prime}$. The northern $\left(\lesssim-5^{\circ} 22^{\prime}\right)$ and the southern $\left(z-5^{\circ} 27^{\prime}\right)$ part of the OMC-1 ridge contains only small clumps.

The ridge does not appear as straight as that seen in the lower resolution observation (Batrla et al. 1983, Hasegawa 1987), and shows the wiggled feature. Though the position angle of the entire OMC-1 ridge is $0-10^{\circ}$, the position angles around the clumps are $20^{\circ}-30^{\circ}$. This means that the clumps have a larger position angle than its mother molecular cloud (the OMC-1 ridge). The polarization observation of $\lambda 1.3 \mathrm{~mm}$ dust continuum emission indicates that the magnetic field has a position angle of $\sim 149^{\circ}$ uniform over the OMC-1 ridge (Leach et al. 1991). The strength of the magnetic field is an order of $1 \mathrm{mG}$. The perpendicular relationship between the magnetic field and the clumps indicates that the magnetic field also plays an important role in the formation of the wiggled structure of the OMC-1 ridge. If we assume that the initial OMC-1 ridge ass straight, a few clumps with a mass of about $100 \mathrm{M} \odot$, and a $1 \mathrm{mG}$ uniform magnetic field with the position angle of $149^{\circ}$, it is possible to make a wiggled ridge in the order of $10^{5}$ years.

\section{Filamentary structures}

Two filamentary structures, $\mathrm{E}$ and $\mathrm{F}$, are dominant in $\mathrm{V}_{\mathrm{LSR}}=7.2-8.6 \mathrm{~km} \mathrm{~s}^{-1}$. Figure II shows the map integrated over the channels. These overall filamentary structures, $\mathrm{E}$ and $\mathrm{F}$, correspond to the E-filament and the $\mathrm{W}$-filament discussed in Murata et al. (1990), respectively. The two filaments in the CS map have different shapes: The E-filament has a length of $200^{\prime \prime}(0.5 \mathrm{pc})$ and a width of $z$ $9^{\prime \prime}-14^{\prime \prime}(0.02-0.03 \mathrm{pc}$ in FWHM $)$ with the position angle of $8^{\circ}$. The ratio of the length to the width is more than 25 . On the other hand, the F-filament is not as narrow as the E-filament. The width is $\sim 14^{\prime \prime}(0.03 \mathrm{pc})$ in the southern part of decl. $=-5^{\circ} 23^{\prime} .5$, and $\sim 23^{\prime \prime}(0.06 \mathrm{pc})$ at decl. $=-5^{\circ} 22^{\prime}$. The F-filament is divided to three parts: the northern part $\left(\right.$ decl. $\left.=-5^{\circ} 21.5^{\prime}-22.5^{\prime}\right)$, the southern part $\left(\right.$ decl. $\left.=-5^{\circ} 23.0^{\prime}-24.0^{\prime}\right)$, and the southern edge $\left(\right.$ decl. $\left.=-5^{\circ} 24.0^{\prime}-24.5^{\prime}\right)$, respectively.

These features seem to be the result of the interaction between the outflow from Orion-KL and the ambient molecular cloud. The evidence for the inter- 


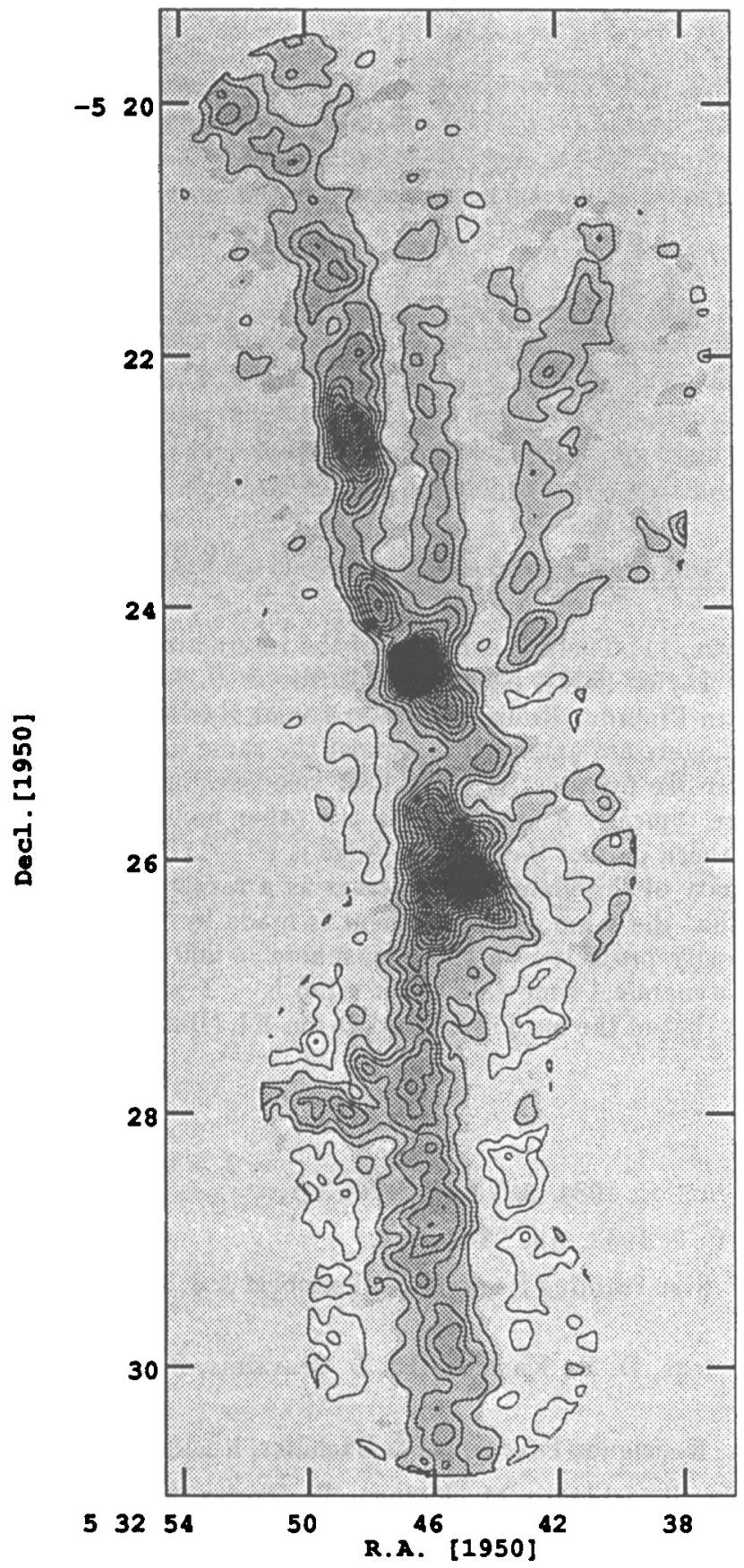

FIGURE I Map of OMC-1 in CS(1-0) line integrated over $\mathrm{V}_{\mathrm{LSR}}=4.1-12.2$ $\mathrm{km} \mathrm{s}^{-1}$. Contour levels are the mean brightness temperature of every $6.8 \mathrm{~K}$ $\mathrm{km} \mathrm{s}^{-1}$. Peak intensity is $187 \mathrm{~K} \mathrm{~km} \mathrm{~s}^{-1}$ at Orion-KL. 


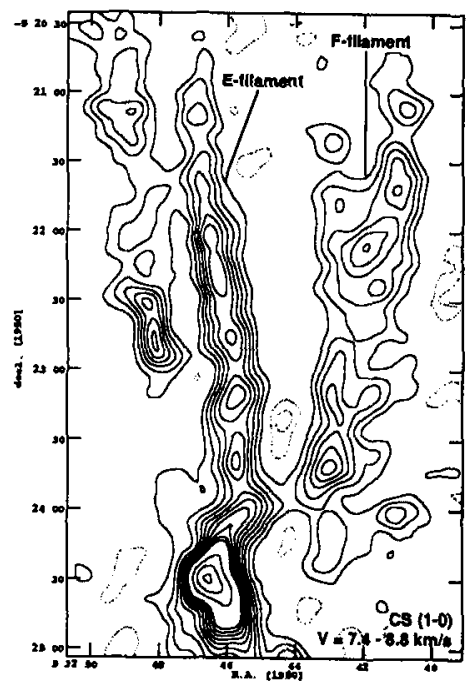

FIGURE II CS (1-0) map of filaments integrated over 7.4$8.8 \mathrm{~km} \mathrm{~s}^{-1}$. Contour levels are every $2.5 \mathrm{~K} \mathrm{~km} \mathrm{~s}^{-1}$ up to 25 $\mathrm{K} \mathrm{km} \mathrm{s}^{-1}$ and every $12.5 \mathrm{~K}$ $\mathrm{km} \mathrm{s}^{-1}$ up to $64 \mathrm{~K} \mathrm{~km} \mathrm{~s}^{-1}$.

actions are as follows. (1) There is evidence of the interaction, such as the $\mathrm{HH}$ objects (Axon and Taylor 1984), $\mathrm{H}_{2}$ fingers (Taylor et al. 1984), and $\mathrm{CO}$ high velocity gas (Martín-Pintado, Rodriguez-Franco, and Bachiller 1990), around the filaments. $\mathrm{H}_{2}$ fingers are extended in almost the same direction as the filaments, and show jet-like features. (2) The LSR velocities of the northern part of the filaments are typically $8 \mathrm{~km} \mathrm{~s}^{-1}$. On the other hand, the typical LSR velocity of the northern part of the OMC-1 ridge is $10-11 \mathrm{~km} \mathrm{~s}^{-1}$. It is likely that the LSR velocity of the filaments decreases as a result of the interaction. (3) If we assume that the $8 \mathrm{~km} \mathrm{~s}^{-1}$ component is made by the pressure of the wind with the velocity projected on the hemisphere $\sim 200 \mathrm{~km} \mathrm{~s}^{-1}$ (Jones and Walker 1985), the dynamical timescale of the wind is $\sim 2 \times 10^{3}$ years, which is almost the same as that of the outflow around Orion-KL (Bally and Lada 1983).

\section{REFERENCES}

Axon, D. J., \& Taylor, K. 1984, MNRAS, 270, 241.

Bally, J., \& Lada, C. J. 1983, ApJ, 265, 824.

Hasegawa, T. 1987, Star Forming Regions, M. Peimbert \& J. Jugaku, Dordrecht: Reidel, 123.

Leach, W. R., Clemens, D. P., Kane, B. D., \& Bravainis, R., 1991, ApJ, 370, 257.

Martín-Pintado, J., Rodriguez-Franco, A., \& Bachiller, R., 1990, ApJ, 357, L49.

Murata, Y., Kawabe, R., Ishiguro, M., Kasuga, T., Morita, K.-I., Takano, T., \& Hasegawa, T. 1990, ApJ, 359, 125.

Taylor, K. N. R., Storey, J. W. V., Sandell, G., Williams, P. M., \& Zealey, W. J. 1984, Nature, 311, 236. 


\section{DISCUSSION}

T. Hasegawa Comment: $\mathrm{CO} J=1-0$ emission observed with the $45 \mathrm{~m}$ telescope shows finger-like structures similar to those seen in CS. The CO fingers appear as shoulders in profiles. In CO J=2-1 observed by Glenn White at JCMT shows wings extending to higher velocities. All these suggest that the finger-like structures are interacting with the outflow.

N. Scoville The linear structure seen in your Orion CS maps might be interpreted as edge-on disks or prolate "cigar" shaped structures. The large number of these structures and their long length suggests that the latter (prolate structures) is more likely. However, this then requires a linear velocity gradient (rigid rotation) in order to avoid winding the structure up. Do you see such velocity gradients?

Y.Murata Yes, we can see linear velocity gradient, about $1 \mathrm{kms}^{-1} / \mathrm{pc}$.

P.T.P. He Comment: There are some evidences of high velocity $\mathrm{CO}$ emission along the filaments, as well as $\mathrm{H}_{2}$ shock emission. 